\title{
Farmland and Crop Quality Improvement for Heavy Metals Based on Six Sigma
}

\author{
Shengping XUE ${ }^{\mathrm{a}, 1}$ and Baohai PAN ${ }^{\mathrm{b}}$ \\ ${ }^{a}$ College of Bioscience and Bioengineering, Hebei University of Economic and \\ Business, Shijiazhuang, China \\ ${ }^{\mathrm{b}}$ Institute of Economics, Hebei Academy of Social Sciences, Shijiazhuang, China
}

\begin{abstract}
Scientific methodology of soil and farmland health management and crop quality improvement is urgent to set up. Our objective was to recognize and sweep away the choke point and improve the farmland health course by applying the philosophy and method of six sigma. Farmland and crop quality improvement for heavy metals belongs to Systems Engineering. SIPOC flux of heavy metals indicates the key process by causal factor analysis through quantitative way. Multi-source and multi-scale data suggest that key process of performance is source control. Measurement processes analyse data and eliminate root causes to improve sigma levels. The study was conducted on farmland and crop health management with concern at the inputs and mechanism that had choke point and high defect rate. DMAIC of sources and exports of cd in the soil will improve and control the Quality of crop and farmland. The premise of implementing six sigma is that the chosen metrics are measurable and effective, but we find a lack of good correlation between terminal index and intermediate index in heavy metal pollution. Suggestion is as follows: Big data could be shared and processed intelligently; policy and culture to pollution-free is more important to farmland and crop health by six sigma.
\end{abstract}

Keywords. Soil and farmland health management, crop quality improvement, heavy metals

\section{Introduction}

Soil quality belongs to the category of Management concepts and natural attributes; most people regard it to same with soil health. The problems about soil environmental quality are mainly discussed at present in soil environmental quality standard, soil environmental quality assessment, soil Environmental quality monitoring, soil pollution control and remediation [1-6]. An International Framework for Evaluating Sustainable Land Management) (FESLM) [7] by Food and Agriculture Organization of the United Nations in 1993 has five pillars as follows: productivity, security, protection, viability acceptability. FESLM is divided into four categories: natural, biological, economic and social indicators. Change our The World: 2030 Agenda for Sustainable Development has got through by The United Nations Sustainable Development Summit meeting endorsed the unity Draft resolution submitted by the General Assembly at its sixty-ninth session. Three dimensions that Economic development, good environment

${ }^{1}$ Corresponding Author, Shengping XUE, College of Bioscience and Bioengineering, Hebei University of Economic and Business, Shijiazhuang, China; Email: xsp6210@163.com. 
and social progress can be measured in SDGs (Sustainable Development Goals) by UNDP (United Nations Development Program) [8].

Heavy metals from industrial waste, and urban household pollution spread widely continue to seep into soil and water. Heavy metals exceeding the limit standard exist in polluted cultivated land for a long time and spread to the surrounding clean environment with water infiltration or water and soil erosion under rainwater scouring, causing groundwater or surface water pollution. Still, others are transferred to agricultural products with the planting and growing process of crops, resulting in crop loss or threatening the health quality of food. Therefore, the impact of cultivated land pollution on food production and health safety is long-term and hidden [9-12].

A system consisting of much processes connecting each other, Defining targets and bound conditions of the system procedure, clear the problem, the analysis process, the need to find out the process, develop evaluation methods and collect relevant data, analyse the root cause of it, find a solution and determine the evaluation items and index system, which is the task of six $\sigma$. Our goal is to improve soil health processes by applying DMAIC methods to identify and remove bottlenecks

\section{Methods and Tools for Soil Quality}

six sigma is an Upgraded version of the quality management system and quantity and process method for solving the problem in a systematic view. Six-sigma can be characterised as a method and a tool to measure quality and to benchmark to progress toward perfection. Six Sigma is a statistical evaluation method, a standard, and more importantly, a management philosophy: it emphasizes the control from the source [13-14]. Like iso9001, ISO 9004 focus customer, and also emphasizes interested party as well as customer. ISO9000 lack a mechanism closely linked to the performance of the enterprise, and is usually assessed by external institutions, the key is not to form an internal code of conduct, learning and continuous improvement of the culture, Six Sigma is a set of management ideas for continuous improvement just like ISO9004. Six Sigma provides a direction, steps and systematic methods for further improvement of enterprises after ISO9001, which not only promotes enterprise reform but also ensures continuous improvement at all levels of soil remediation.

Scientific system and standard of soil environmental quality supervision could be established by six sigma. $3 \sigma-4 \sigma$ level caused by defects need to spend $15-30 \%$ of the sales to make up for later or modification, and the cost of the $6 \sigma$ dropped to $5 \%$ of sales. They are achieving organisational goals by translating strategies and policies into measurable goals at all relevant levels, such as different spatial scale, defining a time frame for each goal and assigning responsibility and authority for achieving the goals, assessing strategic risks and identifying appropriate countermeasures to capture and integrate multi-resources data, providing required resources, identifying relationships between soil quality and farmland quality processes, and carrying out multidimensional and multi-scale activities.

Design products and processes from the perspective of ecological quality, future-oriented process and product of ecological quality are designed at beginning. When improving a process in a threshold state, it is obvious to use DMAIC essentially. Set and achieve goals in a systematic way and demonstrate them. The typical steps of Six Sigma [5]. Improvement Methodology are D-Definition, M- Measurement, A-Analysis, I-Improvement, and C-Control; The typical steps used in Six Sigma 
Design (DFSS) are D-Definition, M-Measurement, A-Analysis, D-Design, V-Validation or I-Identification, D-Design, O-Optimization, and V-Validation. DFSS (Design for Six Sigma DFSS ) does not stop at thinking about processes but takes a higher perspective to design to avoid defects or waste. Make the system flow more reasonable.

Six sigma proceed by Motorola Inc is the seven-step method [15] Choose a problem and describe it clearly; Study the current system; To verify possible causes; Plan and implement solutions; Evaluate the effect; Normalize any valid solution; Reflect on the process and make a plan; Design Improvement Research by Six Sigma. The process of soil quality assessment includes determining soil work able to identify relevant processes and select specific one soil characteristic indexes and the establishment and quantification of soil quality assessment price indices.

\section{Problem and Bottleneck Defined by Six Sigma}

\subsection{Define Phase of DMAIC}

Three points to define the six sigma quality management of soil is as follows:

What is the problem? What are the key processes that affect the problem? Is the process measurable? SIPOC method is applied in process analysis and optimisation at the Define phase.

D-Definition. Identify key processes; The key process plays a role in the key characteristics of the production process, and it has an impact on the quality of the product directly. Pesticide, chemical fertiliser, sewage irrigation and industrial waste disposal pesticides and herbicides may cause excessive arsenic in the soil. The sewage can cause mercury in the soil and car exhaust fumes may cause the excessive lead in soil and other agricultural chemicals (e.g., The excessive application of chemical fertilisers, pesticides, agricultural film, etc.) pollute on a larger scale also.

In the define phase, SIPOC (Supplier Input Process Output Customer) method [16] was used to identify key processes and identify the main problems leading to soil health bottlenecks.

The key process plays a role in the key characteristics of the production process, and it has an impact on the quality of the product directly. At this stage, bottlenecks in various $\mathrm{Cd}$ contamination processes were identified. People and the planet are facing a loss of health. As a result, crop quality often fails to meet the requirements. Therefore, the SIPOC method is used to define the production process of the crop and also to identify processes with bottlenecks, as shown in figure 1 .

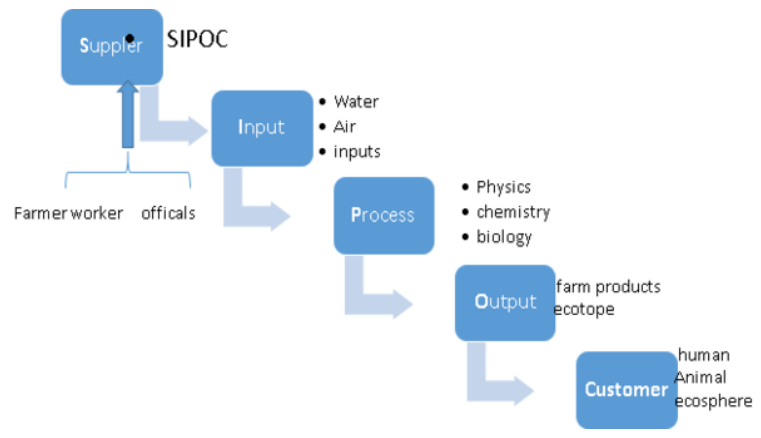

Figure 1. SIPOC of soil health. 
Agricultural source control of water-soil-biological-air mutual pollution exist in cd pollution. As can be seen from the SIPOC method and detailed pollution studies, there are bottlenecks in biological and chemical processes in different pollution processes, as described in Section 3.1.

The major problems identified in biological process and chemical processes.

were overproduction of minerals and industrial products in the pollution process, which resulting in an excess of finished goods inventory leading to wastage. In the biology process of soil, there was an overuse of Agricultural inputs and their low-quality, resulting in bottlenecks. It was also identified that due to the improper variety's layout, there was variation in the production of the country. Lack of process control has led to defects in crop production. In the next phase, the effect of these bottlenecks on the total production of agriculture in terms of value-added and non-value-added activities in the production process was analysed.

Variations in national production were also found due to the improper distribution of varieties. Lack of process control leads to defects in crop production. In the next stage, the impact of these bottlenecks on total agricultural output in terms of value-added and non-value-added activities in the production process is analyzed.

\subsection{Measure Phase of DMAIC}

In the past, the investigation was not combined with the analysis of the source of the soil pollution, leading to the confusion about the cause of soil contamination or soil environmental abnormalities. During the measurement phase, data related to user requirements, consumer voices, and each farm process cycle needs to be recorded. Therefore, in order to recognize added-value and non-added-value events in the pollution process, a vector space model (VSM) was modoled, as shown in figure 2.

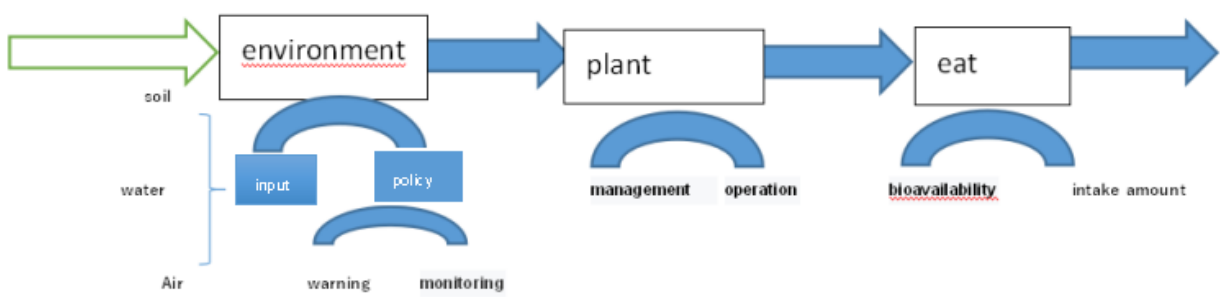

Figure 2. Vector space model (VSM) of heavy metal.

From VSM, non-added-value activities were decided in the pollution remittance. From a particular survey on the pollution cure process of the crop, the value-added activities evaluated were the overuse of input materials for instance fertilizer and pesticide in plant growth and intensify in policy warning and monitoring management.

\subsection{Analysis Phase of DMAIC}

The reaction of heavy metals in multi-phase and multi-media soil was integrated based on the general parameters of the existing database by $\mathrm{Qu}$ et al. [4], the dissolution concentration of heavy metals in soils with different physical and chemical properties could be estimated.

In this stage, the total soil mass caused by the frequent occurrence of defect index in soil is analyzed. Defects [17-19] were found in the CEC, $\mathrm{pH}$ and organic content of the soil, which were caused by underutilization of chemical fertilizers and biological 
pesticides. Index weight of soil in two sites related to crop quality were collected, as shown in table 1.

Table 1. Index weight of soil in two sites of China [17-19].

\begin{tabular}{|c|c|c|c|c|c|c|c|c|c|}
\hline & $\begin{array}{l}\text { Depth of the } \\
\text { barrier layer } \\
\text { from the surface }\end{array}$ & $\begin{array}{l}\text { Profile } \\
\text { configuration }\end{array}$ & $\begin{array}{l}\text { Surface } \\
\text { soil } \\
\text { texture }\end{array}$ & $\begin{array}{l}\text { Organic } \\
\text { content }\end{array}$ & pH & $\begin{array}{l}\text { Salinization } \\
\text { degree }\end{array}$ & $\begin{array}{l}\text { Irrigation } \\
\text { guarantee } \\
\text { rate }\end{array}$ & $\begin{array}{l}\text { Irrigation } \\
\text { water } \\
\text { level }\end{array}$ & $\begin{array}{l}\text { Drainage } \\
\text { condition }\end{array}$ \\
\hline No.1 & 0.03 & 0.08 & 0.07 & 0.04 & 0.06 & 0.12 & 0.30 & 0.06 & 0.24 \\
\hline No.2 & 0.05 & 0.18 & 0.14 & 0.09 & 0.07 & $\mathrm{Nt}$ & 0.27 & $\mathrm{Nt}$ & 0.20 \\
\hline
\end{tabular}

\subsection{Improve Phase of DMAIC}

At this stage, the principal cause analysis was carried out and the dominating reasons for the increase of soil pollution defect level and loss of productivity were found out. Therefore, we used a cause-and-effect diagram to determine the main cause, as shown in figure 3.

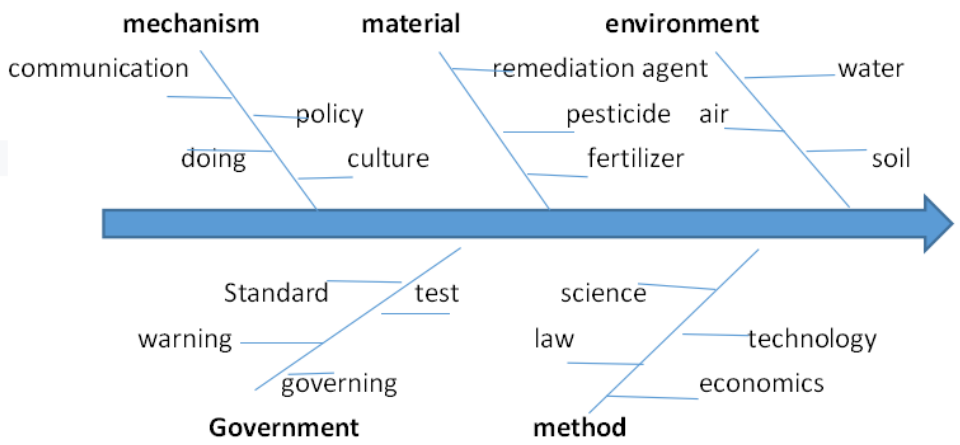

Figure 3. Cause-and-effect diagram of farmland quality.

From a macro perspective, it was identified that the standard for control the crop and repair for the soil because the standard lead activity of people. The root cause for the standard was due to the improper unity index but varies soil. The warning loss to deal with pollution was caused by poor communication and sharing of Information. Effective warning need from a micro perspective, it was determined that the main cause for the "cd rice" was due to more input than output to the grain of rice. The whole chain management includes cut off the source, control the process, and the ends of governance.

The main causes for the cd pollution in the farmland have resulted in defects of crop. There is therefore a need to improve the existing system to reduce the level of losses and defects on farms and in related sectors.

The system evaluation must consist of multiple items and corresponding indexes. Different evaluation items and index systems can be formed according to different evaluation elements emphasised by different systems. Process management is the principle of soil quality management. Soil quality cannot be separated from soil management.

Environmental quality standards for heavy metals have both independence and dependence. Its independence refers to the assignment of absolute criteria values derived from soil background conditions based on natural soil protection or soil quality objectives. At the same time, the dependence means the assignment of relative limits 
based on soil resource management or health and ecological risks.

Attention is focused on a couple of binding targets on the objective, not on non-binding indicators. Soil environmental quality standards now have a few failures: Correlation is not enough to each other. The specification is less, Inadequate adaptability,

Intention is inflexibility, Failure to achieve the purpose of control. Therefore, it is difficult to give a unified national value but to have different specified values depending on different industrial sectors and local administrations in either total amount of extractable heavy metal contents. The pot experiment results unexpectedly found that the content of heavy metals in the active state of soil could not determine the content of heavy metals in the above-ground parts of plants, which indicated that to take the content as the target of soil heavy metals remediation and deviation, it is necessary to develop and establish the mathematical model of multi-source data in the complex soil-water-air-biological system to predict the main key factors [20].

Establish working standards, define management responsibilities and implement process supervision Through continuous improvement to establish process control system and out of control action [21]. Effective technical control of soil pollution rapidly. The program ensures continuity of improvement results. Dynamic quality monitoring platform and visual prediction and early warning system can establish soil environment health from the beginning [22].

The monitoring platform collects and shares information and data on soil pollution more quickly.

Reduct dimensionality, APCA-MLR lock the pollution sources by principal component analysis (PCA), then determined the contribution rate of pollution sources by multiple regression using factor score and pollutant content: $\mathrm{pH}$, organic matter and CEC were the main factors affecting the activity of heavy metals [5].

The relevant model is constructed to realise the analysis and evaluation of soil pollution Price and forecast early warning. The study of soil environmental capacity can modify quality standards for sustainable development of soil, an approach to environmental quality management [23-25].

\subsection{Control Phase of DMAIC}

Keep continuous improvement by Strategic and tactical elements and culture, which encourages continuous learning and improvement. Form a closed loop in one circle of DMAIC and spiral progression by serial DMAIC.

\section{Conclusion}

Farmland and crop quality improvement for heavy metals need composite remediate which are as follows: source control, monitoring and early warning and risk evaulation, technologies and products to remediate, breed resistant varieties of crop, finally ecological remediation. Through flow analysis by quantitative method, this research finds out and solves the key factors step by step. The paper begins with a detailed study of the soil heavy metal pollution. The study was conducted in farmland health management with a focus on the inputs and mechanism which are bottlenecks. Source control of six sigma nips pollution in the bud, low cost and high benefit. Suggestions are as follows: firstly big data of multi-scale and multi-source could be shared and processed intelligently; secondly policy and culture to pollution-free is more important 
to farmland health by six sigma; finally prevention first in different scale and field is necessory for short of remediation method to complex pollution in farmland nowadays.

\section{Acknowledgements}

This work is sponsored by Hebei Provincial Higher Education Teaching Reform Research and Practice Project Gjxgk015 (2017), key project 2016KYZ01 of Hebei University of Economics and Business, and project C2015207019 of the Hebei Province Natural Fund. Thanks PAN Yinjie for pre-reviewing the paper.

\section{References}

[1] Bünemann E K, et al. 2018 Soil quality-A critical review Soil. Boil. Biochem. 120 105-25.

[2] Vilček J and Koco Š 2018 Integrated index of agricultural soil quality in Slovakia J. of Maps 14 68-76.

[3] Liang Z, Gao L, Zhao X, Chen J, Xie Z, Li S, Li R and Yang Z 2019 Assessment of metal pollution, its potential health risks, and origin in different land use types in Zhuhai City, China Arch. Env. C 76 295-307.

[4] Qu X, Xu W, Ren J, Zhao X, Li Y and Gu X 2020 A field study to predict Cd bioaccumulation in a soil-wheat system: Application of a geochemical model J. Hazard Mat. 400 DOI:10.1016/j.jhazmat.2020.123135.

[5] Liu Y, Wen C and Liu X 2013 China's food security soiled by contamination Science 339 1382-1383.

[6] Yang Q, Wu K, Feng Z, Zhao R, Zhang X and Li X 2020 Advancement and revelation of the research on soil quality assessment on large spatial scales Acta Pedologica Sinica 57 565-78.

[7] FAO 1993 An International Framework for Evaluating Sustainable Land Management.

[8] United Nations 2015 Transforming Our World: The 2030 Agenda for Sustainable Development (United Nations General Assembly NewYork).

[9] Youn M, Vries W D and Ros G 2019 Development of a derision support framework to evaluate the impacts of agricultural management on the crop, soil, and environmental quality Wageningen Soil Conference $\mathrm{p} 73$.

[10] Solgi W E, Sheikhzadeh H and Solgi M 2018 Role of irrigation water, inorganic and organic fertilizers in soil and crop contamination by potentially hazardous elements in intensive farming systems: Case study from Moghan agro-industry, Iran J. Geochem. E. 185 74-80.

[11] Yang T, Meng J, Jeyakumar P, Cao T, Liu Z, He T, Cao X, Chen W and Wang H 2020 Stochastic risk assessment of urban soils contaminated by heavy metals in Kazakhstan Sci. Total E. DOI:10.1016/j.scitotenv.2020.141535.

[12] Ramazanova E, Lee S H and Lee W 2020 Source apportionment and source-oriented risk assessment of heavy metals in the sediments of an urban river-lake system Sci. Total E. DOI:10.1016/j.scitotenv.2020.140310.

[13] Juran J M and De Feo J A 2010 Juran's Quality Handbook the Complete Guide to Performance Excellence (McGraw-Hill Professional) p 35.

[14] Yang C 2008 Improving the definition and quantification of quality costs Total Quality Management and Business Excellence 19 75-79.

[15] Marrelli A F 1993 What is your problem? Here is a seven-step method that you can use to analyze and define performance problems Performance 32 1-4 doi.org/10.1002/pfi.4170320703.

[16] Larson W E and Pierce F J 1994 The dynamics of soil quality as a measure of sustainable management in: Doran J W, Coleman D C, Bezdicek D F and Stewart B A (Eds.), Defining Soil Quality for a Sustainable Environment (Madison: Soil Science Society of America Special Publication) pp 37-70.

[17] General Administration of Quality Supervision, Inspection and Quarantine of the People's Republic of China Standardization Administration of China 2016 Cultivated Land Quality Grade GB/T33469-2016 pp 12-30.

[18] General Administration of Quality Supervision, Inspection and Quarantine of the People's Republic of China Standardization Administration of China 2012 Regulation for Gradation on Agriculture Land Quality GB/T28407-2012.

[19] The Ministry of Agriculture of the People's Republic of China 1996 Classification of Type Region NY/T309-1996. 
[20] Xue S and Wang X 2021 Inoculation of soil with cadmium-resistant actinomycetes flora reduces cadmium accumulation in rice (Oryza sativa L.) $I N T . \quad J . \quad E N V . \quad P . \quad R . \quad 9 \quad 1-11$ DOI10.11159ijepr.2021.001.

[21] Olanrewaju F, Uzorh A C and Nnanna I 2019 Lean six sigma methodology and its application in the manufacturing industry-A review Am. J. Mech. Ind. Eng. 4 40-44.

[22] Seybold C A, Hubbs M D and Tyler D D 2002 On-farm tests indicate effects of long term tillage systems on soil quality J. Sustain. Agr. 19 61-73.

[23] Nandakumar N, Saleeshya P G and Harikumar P 2018 Bottleneck identification and process of the scientific committee of the international conference on advances in materials and manufacturing applications ICOnAMMA pp 1217-24.

[24] Cronemyr P 2007 DMAIC and DMADV-differences, similarities and synergies Int. J. Six Sigma and Competitive Advantage 3 193-20.

[25] Zhang L, Cai D, Wang X, Zhang J and Jin K 2005 A study of agricultural tridimension pollution and discussion on its control Agr. Sci. China 4 214-22. 